\title{
Mapeamento Sistêmico de Sensores Reutilizáveis Baseados em Impedância Eletromecânica
}

\section{Systematic Mapping of Reusable Sensors for Impedance-based Health Monitoring}

Bruno Pereira Barella1, Stanley Washington Ferreira de Rezende², Robson Medrado de Oliveira ${ }^{2}$, José dos Reis Vieira de Moura Júnior²

\section{RESUMO}

O método de monitoramento da integridade estrutural baseado em impedância eletromecânica é capaz de identificar mudanças estruturais de modo incipiente. Os transdutores piezelétricos são geralmente utilizados para abstrair as características pertinentes do sistema em análise. Esses transdutores chamados pastilhas piezelétricas (PZTs) são colados à estrutura, exigindo assim um grande número de sensores em todo o sistema. Desta forma, a presente contribuição tem por finalidade realizar um mapeamento sistêmico sobre o tema de sensores reutilizáveis aplicados ao monitoramento de integridade estrutural, tendo como enfoque especial aqueles que empregaram em conjunto o método de impedância eletromecânica. Assim, este trabalho visa propiciar uma base para futuras pesquisas da área, expondo os aspectos relevantes do tema e demonstrando o atual panorama de pesquisas já realizadas. A partir dos resultados observa-se que o tema de sensores reutilizáveis no método de monitoramento da integridade estrutural ainda é um campo pouco explorado, sendo passível de futuras analises.

Palavras-chave: Monitoramento de Integridade Estrutural. Mapeamento Sistêmico. Sensores Reutilizáveis.

\section{ABSTRACT}

The impedance-based structural health monitoring method is able to identify structural changes in an incipient way. Piezoelectric transducers are usually applied in order to abstract the pertinent characteristics of the system under analysis. These transducers called piezoelectric tablets (PZTs) are glued to the structure, thus requiring a large number of sensors throughout the system. Then, the purpose of this contribution is to carry out a systemic mapping on the theme of reusable sensors applied to structural integrity monitoring, with a special focus on those who used the electromechanical impedance method together. Thus, this work aims to provide a basis for future research in the area, exposing the relevant aspects of the theme and demonstrating the current panorama of research already carried out. From the results, it can be seen that the theme of reusable sensors in the structural integrity monitoring method is still a little explored field, being subject to future analysis.

Keywords: Structural Health Monitoring. Systematic Mapping. Reusable Sensors.
Mestrando em Modelagem e Otimização -Unidade Especial de Matemática e Tecnologia Universidade Federal de Goiás brunobarella@hotmail.com

2 Mestrando em Modelagem e Otimização -Unidade Especial de Matemática e Tecnologia Universidade Federal de Goiás stanlev-

washingotn@hotmail.com

3 Mestre em Modelagem e Otimização -Unidade Especial de Matemática e Tecnologia Universidade Federal de Goiás robsonmedradooli@hotmail.co m

Doutor em Engenharia Mecânica -Unidade Especial de Matemática e Tecnologia Universidade Federal de Goiás zereis@ufg.br 


\section{INTRODUÇAOO}

Estruturas ou sistemas mecânicos são utilizados todos os dias por inúmeras pessoas. Estes requerem níveis de segurança adequados para seu funcionamento, porém é inevitável que tais estruturas sofram desgastes ao longo de sua vida útil, gerando assim mudanças nas condições ideais de operação. Segundo Santos et al. (2015), estruturas seguras e duráveis são aquelas bem gerenciadas e monitoradas.

Uma das técnicas consagradas de avaliação da integridade estrutural, com várias aplicações na indústria aeronáutica, tem sido o método de monitoramento baseado em impedância eletromecânica. Nesse método um sensor/atuador piezelétrico é acoplado à estrutura de forma permanente e um circuito monitora a impedância elétrica da pastilha.

Como o transdutor está acoplado à estrutura em estudo, ao ser aplicada uma tensão elétrica sobre ele, o mesmo se deforma gerando uma vibração mecânica no sistema. Esta deformação reflete-se na pastilha PZT, que por sua vez à converte em uma tensão elétrica proporcional.

O acoplamento estrutura/PZT é denominado na literatura por eletromecânico, sendo utilizado como padrão para a investigação de falhas estruturais, de acordo com as modificações periódicas dos sinais medidos (MOURA JR e STEFFEN JR, 2006; MOURA JR, 2008; CHABOWSKI et al., 2015).

Os sensores piezelétricos utilizados para a avaliação estrutural são capazes, a depender das condições de contorno, de detectar alterações em suas propriedades mecânicas em até 2.5 metros de sua origem (HU e YANG, 2007). Desta forma, caracterizando a ferramenta de análise como um método local capaz de identificar danos incipientes.

Aplicações em grande escala geram elevados custos devido à complexidade do ferramental necessário para adquirir os sinais advindos de uma grande rede de sensores, dos quais são responsáveis por monitorar o sistema como um todo. Como exemplo deste problema pode-se destacar o trabalho desenvolvido por Annamdas e Yang (2012), que aplicou uma rede de 30 sensores piezelétricos para o monitoramento de um sistema de suporte de escavação.

Comumente os sensores piezelétricos são acoplados (por colas epóxi) às estruturas a serem analisadas, de modo que este acoplamento não atrapalhe o comportamento dinâmico do sistema (MOURA JR, 2008). No entanto, atualmente técnicas alternativas estão sendo desenvolvidas com o intuito de contornar problemas como grande 
quantidade de sensores para monitoramento de sistemas complexos, não reutilização de sensores após o monitoramento e alto tempo de sensoriamento das estruturas (uma vez que é necessário no mínimo 24 horas para a secagem de alguns tipos de colas).

Este trabalho apresenta um mapeamento sistêmico acerca da utilização de sensores reutilizáveis no monitoramento de integridade estrutural. Para tanto, se é realizada uma breve introdução do método de impedância eletromecânica, explanando os seus aspectos e características aplicadas ao monitoramento de integridade. Em seguida será abordado a metodologia utilizada para desenvolvimento do mapeamento sistêmico, sendo os seus resultados apresentados a posteriori.

\section{IMPEDÁNCIA ELETROMECÂNICA}

As técnicas de monitoramento da integridade estrutural baseadas em análises de assinatura de vibração foram investigadas extensivamente. Esses métodos geralmente envolvem registrar as assinaturas de vibração em uma condição integra e avaliar o estado da estrutura comparando as assinaturas de vibração tomadas em vários momentos durante a vida útil da estrutura. Os dados medidos são processados usando uma variedade de técnicas de processamento de sinais para procurar mudanças nas características que indicam a presença de danos (FARRAR et al., 2003).

O método baseado em análise de assinaturas de vibração geralmente registra assinaturas em uma condição íntegra e avalia o estado da estrutura comparando as assinaturas de vibração tomadas em vários momentos durante sua vida útil. Os dados medidos são processados usando uma variedade de técnicas de processamento de sinais para procurar mudanças nas características que indicam a presença de danos (FARRAR et al., 2003).

O método de monitoramento baseado em impedância eletromecânica é capaz de identificar alterações nas propriedades mecânicas de uma estrutura a partir de pastilhas piezelétricas de titanato zirconato de chumbo, comumente denominadas pastilhas PZT. A partir dos estudos realizados por Liang, Sun e Rogers (1994), sobre a equação da admitância que modela um sistema com acoplamento eletromecânico com 1 grau de liberdade, é possível identificar um dano baseado nas mudanças das características dos parâmetros dinâmicos de uma estrutura. A Equação 1 apresenta a representação da admitância medida numa estrutura de 1 grau de liberdade. 


$$
Y(\omega)=i \omega a\left(\bar{\varepsilon}_{33}^{T}(1-i \delta)-\frac{Z_{s}(\omega)}{Z_{s}(\omega)-Z_{a}(\omega)} d_{3 x}^{2} \hat{Y}_{x x}^{E}\right)
$$

onde $Y(\omega)$ é a admitância elétrica, $Z_{a}$ a impedância mecânica da pastilha $\mathrm{PZT}, Z_{s}$ a impedância mecânica da estrutura, $\hat{Y}_{x x}^{E}$ o módulo complexo de Young do PZT com campo elétrico nulo, $d_{3 x}$ a constante de acoplamento do PZT na direção $x$ a deformação $0, \varepsilon_{33}^{T}$ a constante dielétrica a deformação $0, \delta$ a constante dielétrica do PZT e a geometria constante do PZT.

O processo para obter os sinais de impedância utiliza simultaneamente o efeito direto e inverso. Os sensores utilizados para a coleta das assinaturas e excitação da estrutura em análise são transdutores piezocerâmicos, dos quais podem se destacar o efeito direto onde se submetido a uma deformação mecânica gera um campo elétrico e o efeito inverso no qual se submetido a um campo elétrico gera uma deformação mecânica. Para a aplicação do monitoramento de integridade pelo método da impedância eletromecânica comumente são utilizados uma cadeia de sensores piezelétricos, afim de manter a estrutura monitorada e operante como um todo, como pode ser evidenciado em (ANNAMDAS e YANG, 2012).

Desta forma, para se realizar uma aquisição de sinais deve- se aplicar uma diferença de potencial alternada com uma frequência de excitação alta de modo a obter uma resposta dinâmica da estrutura. Esta resposta dinâmica é coletada pelo sensor e convertida em corrente elétrica e então a mesma é coletada por um sistema de aquisição para então ser processada de modo a retornar a parte real da impedância eletromecânica, uma vez que esta é a mais sensível a alterações nas propriedades mecânicas da estrutura. Assim é possível obter a assinatura de impedância da estrutura a partir da resistência elétrica da pastilha PZT relacionada com a impedância da estrutura (PARK e INMAN, 2005).

A técnica de monitoramento da integridade estrutural baseado em impedância eletromecânica consiste em obter a função resposta em frequência (FRFs) da estrutura. Por meio de alterações na FRF pode-se identificar a presença de uma alteração de parâmetros estruturais que pode ser considerada como dano (RAJU, 1997).

A partir de um sinal obtido por um analisador de impedância realiza-se uma análise qualitativa do dano. Para se obter uma análise quantitativa do dano deve-se aplicar uma métrica de dano. Uma das métricas mais utilizadas é o desvio médio da raiz quadrada (RMSD), proposta por Sun et al. (1995) conforme a Equação 2. 


$$
R M S D=\sqrt{\sum_{i=1}^{n}\left(\frac{\left(\operatorname{Re}\left(Z_{1, i}\right)-\operatorname{Re}\left(Z_{2, i}\right)\right)^{2}}{\operatorname{Re}\left(Z_{1, i}\right)^{2}}\right)}
$$

Na Equação 2 tem-se que $\operatorname{Re}\left(Z_{1, i}\right)$ é a parte real da impedância do sinal da estrutura sem danos (baseline), $\operatorname{Re}\left(Z_{1, i}\right)$ é a parte real da impedância do sinal da estrutura com dano. A partir da Equação 2 pode-se realizar uma análise quantitativa, ou seja, mensurar o dano ocorrido na estrutura comparando-o com o estado inicial.

\section{METODOLOGIA}

Estudos de mapeamento sistemático ou estudos de escopo são projetados para dar uma visão geral de uma área de pesquisa através da classificação e avaliação das contribuições feitas até o momento (PETERSEN et al., 2008; PETERSEN, VAKKALANKA e KUZNIARZ, 2015). Dessa forma, esses mapeamentos tem por finalidade proporcionar uma nova gama de ideias, demonstrando vertentes que ainda não foram verificadas (JUNIOR, QUEIROZ e LIMA, 2018).

A partir do exposto, as fases de elaboração deste artigo de revisão se fundamentaram na metodologia de mapeamento de pesquisas proposta por Bailey et al. (2007) e Viana, Formoso e Kalsaas (2012). Essa por si, consiste do levantamento sistemático da literatura que se conjectura em uma estrutura de busca por periódicos, mineração e análise de dados (BAILEY et al., 2007).

A revisão sistemática da literatura, também conhecida como mapping study, permite identificar a consistência de estudos existentes sobre determinado assunto, porém não se limitando apenas a reprodução dos resultados (COOPER, 2016). Assim sendo, o mapeamento realizado neste trabalho se baseou na pesquisa em bases de dados que possuem uma vasta quantidade de publicações e ferramentas de buscas, as quais, possibilita o uso de expressões-chave para a seleção dos artigos.

Utilizou-se ao total quatro bases de dados, a saber: Scopus, Web of Science, Engineering Village e Portal de Periódicos da Coordenação de Aperfeiçoamento de Pessoal de Nível Superior (CAPES). Embora existam outras bases de dados, a pesquisa limitou-se somente a essas, visto que elas já são capazes de contemplar um número significativo de artigos e periódicos.

As expressões lógicas empregadas foram: (a) "reusable piezoelectric" AND "Electromechanical impedance" e (b) "reusable PZT pacth" AND "Health monitoring". Foram escolhidas estas palavras chave pois abrange a aplicação dos sensores 
reutilizáveis tanto em técnicas de monitoramento de integridade estrutural quanto em um dos métodos mais utilizados, o baseado em impedância eletromecânica.

Foram aplicados filtros como: idioma (inglês); tipo de publicação (artigo de periódico revisado por pares) e por fim não se aplicou restrições por área de conhecimento. Ordenou- se os artigos por ano de publicação para então dar início a seleção dos mesmos filtrando assim em cada base de dados os artigos por título.

O critério empregado para a seleção dos artigos durante a filtragem por título em cada base de dados utilizada, foram se os mesmos continham as palavras chaves utilizadas na pesquisa e se o tema do artigo se relacionava com o tema estudado, que nesse caso é da utilização de sensores reutilizáveis no monitoramento de integridade estrutural. Sendo assim trabalhos que apresentavam divergência entre o tema estudado e o tema apresentado pelo título foram descartados.

Em seguida, foi montada uma planilha contendo os artigos selecionados separados por base de dados e por pesquisa. Foram analisadas as repetições dos artigos na mesma base de dados e em base de dados diferentes. Esta filtragem proporcionou selecionar os artigos que terão os resumos lidos para realização da filtragem final.

De posse dos artigos sem repetição foi realizada a leitura dos resumos analisando a aderência do tema discorrido nos artigos em comparação com o tema estudado. Ao finalizar esta etapa, os artigos foram filtrados e salvos para a leitura completa. Durante a leitura dos artigos selecionados foi empregada a técnica snowball sampling, em busca de artigos de referência na área e que por ventura não tenham sido identificados ou selecionados no mapeamento sistemático (PAULA et al., 2017).

\section{DISCUSSÃO E RESULTADOS}

\subsection{MAPEAMENTO SISTÊMICO}

Foram organizados os artigos em forma de tabela com o intuito de organizar e tornar mais claro os procedimentos para o emprego do mapeamento sistêmico. A Tabela 1 apresenta os resultados totais obtidos.

Na Tabela 1 é possível identificar as pesquisas "reusable PZT pacth" AND "Health monitoring" denominada "P1" e "reusable piezoelectric" AND "Electromechanical impedance" denominada "P2". Também pode-se observar a relevância do procedimento de filtragem onde inicialmente as pesquisas forneceram cerca de 75 artigos, sendo filtrados por título para 40, e por fim, com a leitura dos resumos para 10 artigos. Com a 
leitura dos artigos pode-se identificar 2 trabalhos que não foram encontrados através das pesquisas nas bases de dados, isto devido à falta da utilização das palavras chaves nos títulos e resumos dos artigos encontrados.

Tabela 1: Dados do mapeamento sobre o tema sensores reutilizáveis

\begin{tabular}{|c|c|c|c|c|c|}
\hline \multirow{2}{*}{ Banco de Dados } & \multicolumn{2}{|c|}{ Quantidade } & \multicolumn{2}{|c|}{ Filtragem Título } & \multirow[b]{2}{*}{ Resumo } \\
\hline & $\mathrm{P} 1$ & $\mathrm{P} 2$ & $\mathrm{P} 1$ & $\mathrm{P} 2$ & \\
\hline CAPES & 33 & 17 & 11 & 4 & 9 \\
\hline Scopus & 3 & 5 & 3 & 5 & 1 \\
\hline Web of Science & 3 & 5 & 3 & 5 & 0 \\
\hline Engineering Village & 3 & 6 & 3 & 6 & 0 \\
\hline Subtotal & 42 & 33 & 20 & 20 & \\
\hline Total & \multicolumn{2}{|c|}{75} & \multicolumn{2}{|c|}{40} & 10 \\
\hline \multicolumn{5}{|c|}{ Total após snowball sampling } & 12 \\
\hline
\end{tabular}

Fonte: Elaborada pelos autores.

Por se tratar de um tema relativamente novo, os artigos selecionados para leitura completa foram publicados entre 2009 e 2018. A Figura 1 apresenta a quantidade de artigos publicados por ano.

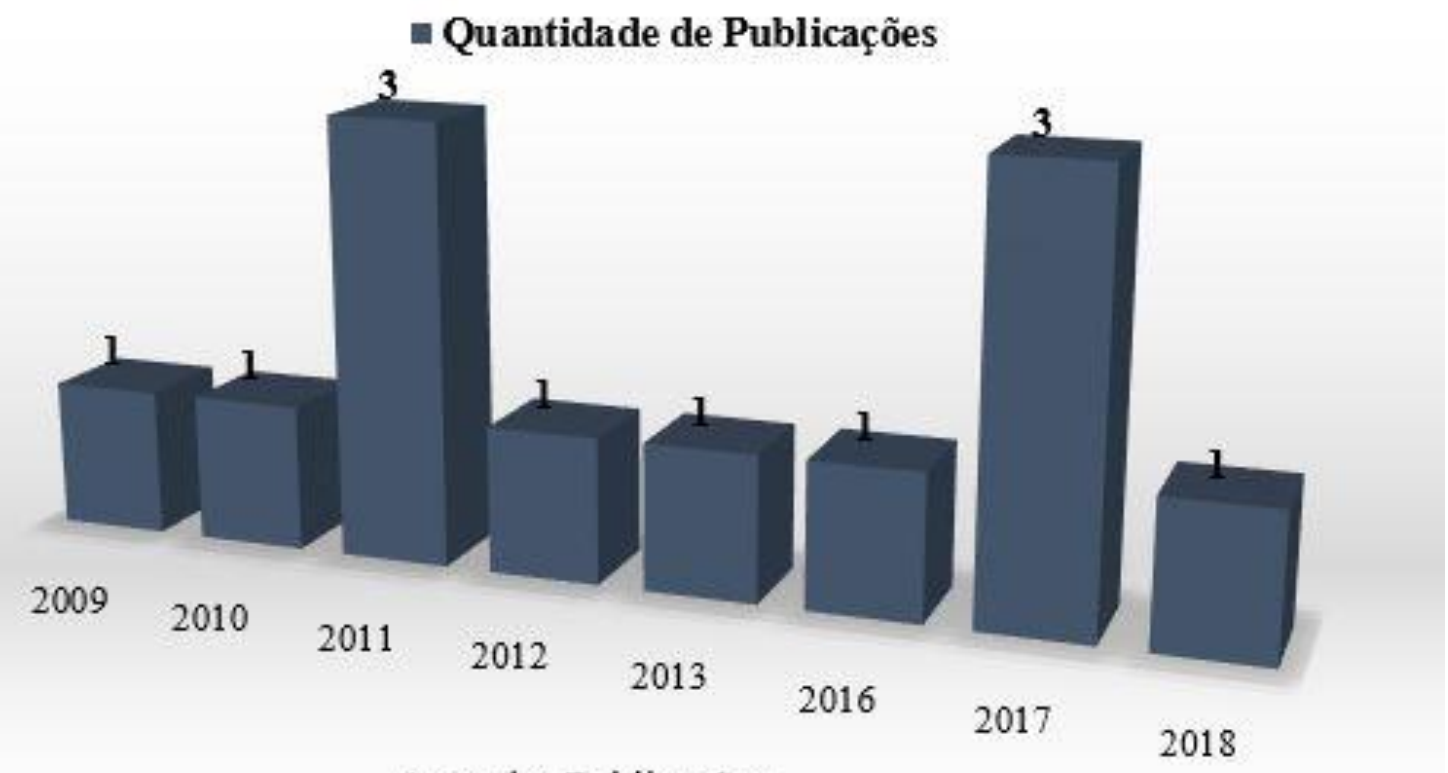

Anos das Publicações

Figura 1. Quantidade de publicações por ano

(Fonte: 0 autor) 
Os periódicos que possuem a maior quantidade de artigos selecionados no mapeamento estão dispostos na Tabela 2. Os periódicos Smart Materials and Structures, Sensors, The International Society for Optical Engineering e Jornal of Intelligent Material Systems and Structures foram os que apresentaram o maior número de publicações, com 2 artigos publicados.

Tabela 2: Principais periódicos com artigos publicados sobre o tema

\begin{tabular}{|c|c|}
\hline Periódicos & $\begin{array}{c}\text { Quantidade de Artigos } \\
\text { Publicados }\end{array}$ \\
\hline Smart Materials and Structures & 2 \\
\hline $\begin{array}{c}\text { Proceedings of SPIE - The International Society for } \\
\text { Optical Engineering }\end{array}$ & 2 \\
\hline Sensors & 2 \\
\hline Journal of Intelligent Material Systems and Structures & 1 \\
\hline Journal of Sensors & 1 \\
\hline Smart Materials Research & 1 \\
\hline Procedia Engineering & 1 \\
\hline Applied Sciences & 2 \\
\hline
\end{tabular}

Fonte: Elaborada pelos autores.

Os pesquisadores que realizaram publicações acerca do tema são apresentados pela Tabela 3. Nesta é possível identificar os autores que mais publicaram artigos sobre o tema estudado.

Tabela 3: Principais autores de artigos sobre o tema sensores reutilizáveis

\begin{tabular}{|c|c|c|}
\hline Pesquisador & $\begin{array}{c}\text { Quantidade de Artigos } \\
\text { Publicados }\end{array}$ & Data de Publicação \\
\hline Silveira, Ricardo Zanni Mendes & 1 & 2017 \\
\hline Divsholi, Bahador & 2 & 2009 e 2011 \\
\hline Na, Wongi S. & 5 & $2016,2017^{2}$ e 2018 \\
\hline Tawie, R. & 1 & 2010 \\
\hline Yang, Yaowen & 1 & 2011 e 2013 \\
\hline Providakis, C. & 2 & 2012,2018 \\
\hline
\end{tabular}

Fonte: Elaborada pelos autores.

Foram classificados os artigos selecionados quanto à vertente de monitoramento empregada. A Figura 2 apresenta as vertentes encontradas com a respectiva quantidade 
de artigos classificados. Este gráfico traz o foco atual da área de pesquisa, explicitando quais as vertentes mais visadas pelos pesquisadores de sensores reutilizáveis aplicados ao monitoramento de integridade estrutural.

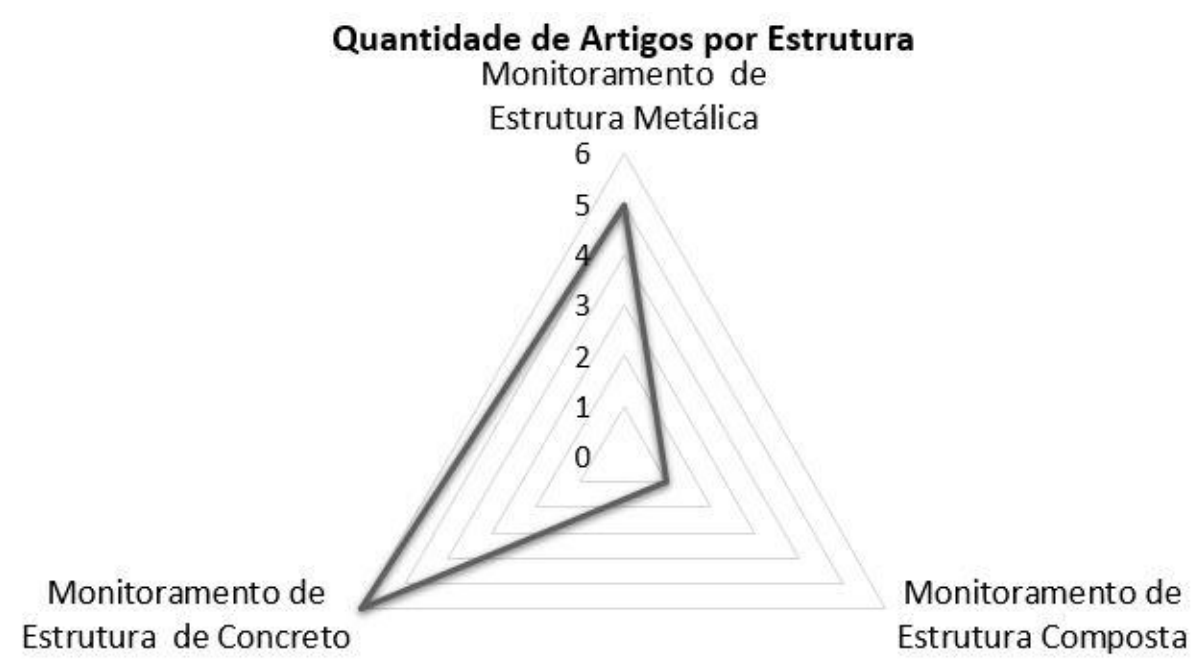

Figura 2. Quantidade de publicações por vertente

(Fonte: o autor)

\subsection{CONTRIBUIÇÕES DE MONITORAMENTO DE ESTRUTURAS COMPOSTAS}

A vertente da aplicação dos sensores reutilizáveis no monitoramento de estruturas compostas, onde foi encontrado um artigo desenvolvido por $\mathrm{Na}$, Tawie e Lee (2012) que utiliza 18 corpos de prova criados por meio da união de duas placas de compósito de fibra de vidro epóxi juntamente com três placas de alumínio foram preparadas para testes adicionais. Ele também realizou experimentos para avaliar a espessura de sensores reutilizáveis em comparação com sensores colados com cola epóxi. Também avaliou a capacidade de detecção de dano utilizando sensores reutilizáveis. Foram constatados que com uma escolha adequada da faixa de frequência (região ressonante da pastilha piezelétrica livre) é possível detectar danos em estruturas compostas sujeitas a desgastes.

\subsection{CONTRIBUIÇÕES DE MONITORAMENTO DE ESTRUTURAS METÁLICAS}

Para a vertente da aplicação dos sensores reutilizáveis no monitoramento de estruturas metálicas foram selecionados 5 artigos. Uma destas contribuições foi a desenvolvida por Silveira, Campeiro e Baptista (2017), onde o principal objetivo foi 
apresentar três tipos de montagem para avaliação da integridade estrutural baseado em impedância eletromecânica, são elas: montagem magnética (imã), montagem baseada em fio de metal e montagem convencional usando cola epóxi. Utiliza o teste Pencil-lead breaks (PLB test) para validar a sensibilidade das pastilhas PZT em cada uma das montagens. Segundo o teste realizado, é esperado uma menor sensibilidade ao utilizar a montagem magnética e uma maior com a montagem convencional. Segundo Silveira, Campeiro e Baptista (2017), as três montagens são capazes de abstrair as características do sistema mecânico, ou seja, são capazes de identificar um dano. Porém, o método convencional fornece uma maior sensibilidade.

$\mathrm{Na}$ e Baek (2017) também desenvolveu um trabalho onde o principal objetivo foi apresentar um experimento para verificação da redução de espessura utilizando placas metálicas. Investigaram o problema da reconexão do imã na estrutura e o solucionou usando uma rede PNN (Rede Neural Probabilística). A princípio, realizou 10 coletas em um experimento utilizando placas de metal colando e retirando o imã a cada coleta de assinatura. Ele constatou que existe uma variação nas assinaturas de impedância ao realizar o experimento, ou seja, é necessário distinguir as alterações de assinatura de impedância de uma estrutura danificada das alterações de assinatura causadas pela reconexão, assim, justificando o uso da rede PNN. Também foram realizados mais 2 experimentos: um utilizando placas de metal de $0.15 \mathrm{~mm}$ de espessura e outro com $0.3 \mathrm{~mm}$. Para ambos, o procedimento foi remover uma a uma das 5 placas sobrepostas e descolar e colar o PZT a cada remoção de placa. As assinaturas deste procedimento foram utilizadas para treinamento da rede uma vez que estas variam aleatoriamente prejudicando a identificação de falha.

$\mathrm{Na}$ (2016) realizou um estudo onde avaliou o desempenho do dispositivo piezelétrico reutilizável (sensor PZT) para estruturas metálicas sendo examinado em dois tipos diferentes de cenários de danos progressivos. No geral, o dispositivo PZT reutilizável mostrado neste estudo identificou com sucesso os danos com a possibilidade de detecção de perda de peso.

Na e Baek (2016) apresenta uma técnica utilizando um veículo aéreo não tripulado (UAVs) drone para a inspeção da integridade estrutural, de modo a eliminar a necessidade da utilização de um grande número de sensores ao longo da estrutura sobe análise. Ele verificou a influência da vibração do drone nas coletas das assinaturas e constatou que esta era mínima. Analisou a repetibilidade das assinaturas de impedância 
realizando a remoção e acoplamento do sensor manualmente e automaticamente por meio do drone. Foi constatado pela aplicação da métrica RMSD que a manual foi mais assertiva com $3.5 \%$ de erro enquanto que o drone teve $7 \%$. Foi realizado um experimento destrutivo de dano progressivo onde em uma placa metálica aplicou 20 cortes de $1 \mathrm{~mm}$ de profundidade. Nele foi possível identificar os danos, porém, todos os valores de métrica possuem valores próximos, constatando uma baixa sensibilidade ao dano proposto. Também foi realizado um experimento para avaliação da variação da espessura por meio de placas metálicas sobrepostas unidas pela forca magnética do imã. Foi constatada uma baixa variação das assinaturas, porém, a métrica apresentou bons resultados aumentando o índice durante a remoção de cada placa.

$\mathrm{Na}$ et al. (2018) desenvolveu uma contribuição buscando relacionar as métricas de dano mais utilizadas no monitoramento de integridade estrutural baseado em impedância eletromecânica com danos propostos e variações na amplitude das assinaturas de impedância. Foi identificado que a métrica MAPD (Mean Absolute Percentage Deviation) teve o melhor desempenho com amplitudes maiores nas assinaturas de impedância. $A$ métrica CCD (Correlation Coefficient Deviation) apresentou o melhor resultado com pequenas amplitudes nas assinaturas.

\subsection{CONTRIBUIÇÕES DE MONITORAMENTO DE ESTRUTURAS DE CONCRETO}

Foram utilizados os sensores piezelétricos reutilizáveis tanto para monitorar danos nas estruturas do concreto quanto para monitorar hidratação, ganho de resistência e taxa de hidratação devido ao uso de retardadores.

Divsholi e Yang (2009) desenvolveram um trabalho onde monitorou a hidratação inicial do concreto através de uma pastilha PZT colada a um pedaço de metal com dois parafusos apertados dentro de furos feitos no metal. Os parafusos e a superfície do metal penetram parte do concreto fresco. Com o desenvolvimento de experimentos constatou que pastilhas piezelétricas mais espessas são mais sensíveis quando comparadas com menos espessas. Também constatou a capacidade de monitorar o ganho de fora e taxa de hidratação do concreto com amostras variando o uso de retardadores.

Divsholi e Yang (2011) apresentam uma contribuição utilizando pastilhas piezelétricas coladas na superfície de um corpo de prova de concreto, inserindo os sensores nos corpos de prova e aplicando sensores piezelétricos reutilizáveis. Foi constatado que os sensores incorporados ao corpo de prova são mais sensíveis para 
monitoramento de hidratação quando comparados com os reutilizáveis. Porém, relata que é possível monitorar a hidratação e detecção de dano com os sensores reutilizáveis.

Tawie e Lee (2011) realizaram experimentos para avaliar mudanças de umidade em uma amostra de argamassa de cimento e o tempo necessário para a secagem completa a partir de sensor reutilizável fixado dentro do corpo de prova com o auxílio de uma haste metálica. Os resultados experimentais do monitoramento de danos usando o sensor reutilizável mostram que a configuração reutilizável proposta é sensível a danos internos devido a inserção da haste dentro da argamassa.

Yang, Divsholi e Soh (2010) propuseram um trabalho, onde uma configuração da pastilha PZT reutilizável para monitorar a hidra- tação inicial do concreto e a saúde estrutural é desenvolvida. Assim, um pedaço da pastilha PZT é colado a um invólucro com dois parafusos apertados dentro dos orifícios perfurados no invólucro. A configuração da pastilha PZT reutilizável desenvolvida neste trabalho é capaz de monitorar efetivamente a hidratação inicial do concreto e pode ser usada para SHM. Com uma camada de ligação controlada, a repetibilidade das assinaturas da pastilha PZT foi confirmada e, portanto, a confiabilidade da configuração reutilizável. Descobriu-se também que as pastilhas PZTs mais espessas apresentam maior sensibilidade quando comparados as mais finas.

Providakis e Liarakos (2011) e Providakis, Liarakos e Kampianakis (2013) utilizam sistema ativo de sensoriamento sem fio que consiste de um circuito de medição de impedância eletromecânico miniaturizado e um transdutor piezelétrico reutilizável apropriadamente instalado em um gabinete confeccionado em Teflon para monitorar o desenvolvimento da resistência do concreto em idades iniciais e estados de hidratação inicial.

\section{CONSIDERACÕES FINAIS}

Foram encontradas três vertentes de aplicação dos sensores reutilizáveis, são elas: estruturas de concretos, estruturas metálicas e estruturas compostas. Nessas vertentes foram analisados os trabalhos encontrados no mapeamento de modo a responder as perguntas elaboradas inicialmente.

Com o emprego do mapeamento sistêmico foi possível identificar uma maior utilização dos sensores piezelétricos reutilizáveis no monitoramento de estruturas de concreto, no qual se destacam a utilização para monitorar a hidratação do concreto, 
avaliação da espessura do sensor utilizado, detecção de danos internos, ganho de força e o desenvolvimento de resistência do concreto em idades variadas.

A segunda vertente mais utilizada foi a do emprego dos sensores reutilizáveis em estruturas metálicas, das quais se destacam comparações entre tipos diferentes de sensoriamento de estruturas, avaliar a redução de espessura e reconexão do sensor reutilizável quando se utiliza imãs, análise de tipos de dano progressivo utilizando sensores reutilizáveis, emprego de drone para monitoramento de grandes áreas e por fim comparação das métricas de dano mais comuns.

E finalmente a vertente onde se emprega o monitoramento de materiais compostos, onde avaliam a degradação da colagem de duas placas de compósito de fibra de vidro e também realizam comparação do sensoriamento convencional (colado) e o sensoriamento reutilizável.

Em se tratando da qualidade e confiabilidade dos sensores, os mesmos reutilizáveis avaliados não foram superiores aos convencionais em nenhum caso.

Concluindo, através do mapeamento realizado neste trabalho é possível identificar lacunas para o desenvolvimento de novas pesquisas. Além disso esta revisão busca direcionar as publicações mais relevantes para o tema estudado.

\section{REFERÉNCIAS}

ANNAMDAS, V. G. M. e YANG, Y. Practical implementation of piezo-impedance sensors in monitoring of excavation support structures. Structural Control and Health

Monitoring, v. 19, n. 2, p. 231-245, 2012.

BAILEY, J. et al. Evidence relating to object-oriented software design: A survey. First International Symposium on Empirical Software Engineering and Measurement. IEEE, p. 482-484, 2007.

CHABOWSKI, K. et al. Simple wide frequency range impedance meter based on AD5933 integrated circuit. Metrology and Measurement Systems, v. 22, n. 1, p. 13-24, 2015.

COOPER, I. D. "What is a mapping study?". Journal of the Medical Library Association: JMLA, Medical Library Association, v. 104, n. 1, p. 76, 2016.

DIVSHOLI, B. S. e YANG, Y. Application of reusable PZT sensors for monitoring initial hydration of concrete. Sensors and Smart Structures Technologies for Civil, Mechanical, 
and Aerospace Systems, International Society for Optics and Photonics, 7292, 729222, 2009.

DIVSHOLI, B. S. e YANG, Y. Comparison of embedded, surface bonded and reusable piezoelectric transducers for monitoring of concrete structures. Sensors and Smart Structures Technologies for Civil, Mechanical, and Aerospace Systems, International Society for Optics and Photonics, 7981, 798151, 2011.

FARRAR, C. et al. Over-view of piezoelectric impedance-based health monitoring and path forward. Shock and Vibration Digest, v. 35, n. 6, p. 451-463, 2003.

HU, Y. e YANG, Y. Wave propagation modeling of the PZT sensing region for structural health monitoring. Smart Materials and Structures, v. 16, n. 3, p. 706, 2007.

JUNIOR, J. E. B., QUEIROZ, P. G. G. e LIMA, R. W. A study of the publications of educational robotics: A systematic review of literature. IEEE Latin America Transactions, v. 16, n. 4, p. 1193-1199, 2018.

LIANG, C.; SUN, F. P. e ROGERS, C. A. Coupled Electromechanical Analysis of Adaptive Material System-determination of Actuator Power Consumption and System Energy Transfer. Journal of Intelligent Material Systems and Structures, v. 5, p. 21-20, 1994.

MOURA JR, J. D. R. V. e STEFFEN JR, V. Impedance- based health monitoring for aeronautic structures using statistical meta-modeling. Journal of Intelligent Material Systems and Structures, v. 17, n. 11, p. 1023-1036, 2006.

\section{MOURA JR, J. D. R. V. Uma contribuição aos sistemas de monitoramento de} integridade estrutural aplicada a estruturas aeronáuticas e espaciais. Tese (Doutorado em Engenharias) - Universidade Federal de Uberlândia, Uberlândia, 2008.

NA, W. S. Progressive damage detection using the reusable electromechanical impedance method for metal structures with a possibility of weight loss identification. Smart Materials and Structures, v. 25, n. 5, p. 055039, 2016.

NA, W. S. et al. Effects of applying different resonance amplitude on the performance of the impedance-based health monitoring technique subjected to damage. Sensors, v. 18, n. 7, p. 2267, 2018. 
NA, W. S. e BAEK, J. Impedance-based non- destructive testing method combined with unmanned aerial vehicle for structural health monitoring of civil infrastructures. Applied Sciences, v. 7, n. 1, p. 15, 2016.

NA, W. S. e BAEK, J. Impedance based health monitoring technique with probabilistic neural network for possible wall thinning detection of metal structures. Sensors, 2017.

NA, S.; TAWIE, R. e LEE, H. K. Electromechanical impedance method of fiber-reinforced plastic adhesive joints in corrosive environment using a reusable piezoelectric device. Journal of Intelligent Material Systems and Structures, v. 23, n. 7, p. 737-747, 2012.

PARK, G. e INMAN, D. J. Impedance-based structural health monitoring. Damage Prognosis for Aerospace, Civil and Mechanical Systems, p. 275-292, 2005.

PAULA, H. M. et al. Mapeamento sistemático de referências do uso do BIM na compatibilização de projetos na construção civil. REEC - Revista Eletrônica de Engenharia Civil, v. 13, n. 1, 2017.

PETERSEN, K. et al. Systematic mapping studies in software engineering. EASE, v. 8, p. 68-77, 2008.

PETERSEN, K.; VAKKALANKA, S. e KUZNIARZ, L. Guide-lines for conducting systematic mapping studies in software engineering: An update. Information and Software Technology, v. 64, p. 1-18, 2015.

PROVIDAKIS, C. e LIARAKOS, E. T-wieye: An early-age concrete strength development monitoring and miniaturized wireless impedance sensing system. Procedia Engineering, v. 10, p. 484-489, 2011.

PROVIDAKIS, C.; LIARAKOS, E. e KAMPIANAKIS, E. Nondestructive wireless monitoring of early-age concrete strength gain using an innovative electromechanical impedance sensing system. Smart Materials Research, 2013.

RAJU, V. Implementing impedance-based health monitoring. Tese (Doutorado em Engenharias), Virginia Tech. 1997. 
SANTOS, A. D. F, et al. Data management system for structural health monitoring. IEEE Latin America Transactions, v. 13, n. 4, p. 1090-1097, 2015.

SILVEIRA, R. Z. M.; CAMPEIRO, L. M. e BAPTISTA, F. G. Performance of three transducer mounting methods in impedance-based structural health monitoring applications. Journal of Intelligent Material Systems and Structures, v. 28, n. 17, p. 2349-2362, 2017.

SUN, F. P. et al. Truss structure integrity identification using PZT sensor-actuator. Journal of Intelligent material systems and structures, v. 6, n. 1, p. 134-139, 1995.

TAWIE, R. e LEE, H. K. Characterization of cement-based materials using a reusable piezoelectric impedance-based sensor. Smart Materials and Structures, v. 20, n. 8, p. $085023,2011$.

VIANA, D. D.; FORMOSO, C. T. e KALSAAS, B. T. Waste in construction: a systematic literature review on empirical studies. 20th Annual Conference of the International Group for Lean Construction, San Diego, USA, p. 18-20, 2012.

YANG, Y.; DIVSHOLI, B. S. e SOH, C. K. A reusable PZT transducer for monitoring initial hydration and structural health of concrete. Sensors, v. 10, n. 5, p. 5193-5208, 2010. 